\title{
Kulturelle Identität und die Metapher von den gepackten Koffern
}

\section{Ein Rückblick und eine Vorschau}

„Juden in Deutschland“ und deren „gepackte Koffer“, auf denen sie seit 1945 sitzen - ist diese Metapher noch immer aktuell? Halten die Juden, die nach 1945 in den DP-Camps auf ihre Ausreise warteten, dann aber doch in Deutschland blieben, ihre Koffer noch immer gepackt? Ebenso die wenigen deutschen Juden, die im Versteck überlebten oder aus der Emigration nach Deutschland zurückkehrten, wie steht es um deren Koffer? Und diejenigen, die nach 1989 die ehemalige Sowjetunion gen Westen verließen, haben sie ihre Koffer in Berlin, Frankfurt oder München schon ausgepackt? Und wenn wir bei der Metapher des Koffers bleiben wollen: was war bzw. ist drin in den Koffern?

Als die deutschsprachigen Juden nach 1933 ihr Heimatland verließen, waren die Koffer mit Fotoalben und weißen bügelglatten Tischdecken, aber ebenso mit Werken von Goethe, Schiller oder Eichendorff gefüllt - all diese Dinge sollten ihnen in der Fremde ein Stück Heimat geben.

Aber was hatten die ehemaligen osteuropäischen DPs, die Deutschland nur als Zwischenstopp auf dem Weg nach Amerika oder Israel ansahen und dann doch dort blieben, Kinder bekamen und sich irgendwann auch häuslich einrichteten - trotzdem aber den gepackten Koffer immer in Sichtweite, darin? Und was befand sich in den Koffern, die in Odessa, Wolgograd oder Moskau gepackt und auf die Reise nach Berlin, Frankfurt oder München mitgenommen wurden? Wenn es auch Bücher waren, um welche Autoren und welche Themen handelte es sich?

Wo verorten sie alle sich, welche Identitäten sind ihnen eigen, welches Selbstverständnis zeichnet sie aus, was verbinden sie mit Heimat und in welcher Kultur fühlen sie sich zuhause? Und gibt es ein verbindendes Element zwischen den auf 200.000 bis 300.000 geschätzten, heute in Deutschland lebenden Juden, von denen etwa 110.000 Mitglieder in den Jüdischen Gemeinden sind? Es handelt sich dabei um die Nachfahren unterschiedlicher Gruppen, die das jüdische Leben nach 1945 repräsentierten: Da waren zunächst die, die in Deutschland im Versteck (ca. 3.000) oder als „Nicht-arischer“ Ehepartner (ca. 12.000) sowie die Konzentrationslager überlebt hatten (ca. 8.000), oder die Remigranten die aus dem Exil nach Deutschland zurückkehrten (schätzungsweise $5 \%$ der aus Deutschland geflohenen Juden), sei es aus politischen Gründen oder des Heimwehs wegen. Diese beiden Gruppen stellten seit den 1950er Jahren die Minderheit der Juden 
in Deutschland dar. Weit größer war die Gruppe der osteuropäischen DPs, die die Konzentrationslager überlebt hatten, meist als einzige der Familie. Die Mehrzahl von ihnen stammte aus Polen und Ungarn. Ihre traumatischen Erfahrungen des Holocaust belasteten die Sicht auf das Nachkriegsdeutschland nicht minder als das fremde Land, die eher feindselige Umwelt, die fremde Sprache und die fremde Kultur, die den ohnedies schon schweren Alltag prägten. Um ihre Jugend gebracht, die Familie verloren und nicht selten ohne Schul- und Ausbildung, schien ein Neuanfang fast aussichtslos. Und doch war der Wille zum Weiterleben ungebrochen - trotz aller Hemmnisse und Hürden. Selbst die schroffe Kritik von Juden aus aller Welt - „Wie kann ein Jude nach dem Holocaust im Land der Täter leben?“ - hinderte die erste Generation - ob osteuropäische DPs oder Rückkehrer aus dem Exil - nicht daran, sich in einem Leben im Nachkriegsdeutschland einzurichten, Kinder in die Welt zu setzen, neue Gemeindestrukturen aufzubauen und allen negativen Prophezeiungen zu trotzen. Aber was bedeutete diese Entscheidung der Elterngeneration für die Kinder? Die Hypothek der zweiten Generation, die den Entschluss der Eltern in Deutschland zu leben hinnehmen musste, war für nicht wenige von ihnen immens. Paul Spiegel, 2002-2006 Vorsitzenden des Zentralrats der Juden in Deutschland, bemerkte einmal als Vertreter dieser zweiten Generation: „Ich gebe auch zu, wenn ich zu Kriegsende vielleicht 25 oder 30 und nicht acht Jahre alt gewesen wäre, ich wäre nicht nach Deutschland zurück gegangen. “1 Es ist durchaus nachvollziehbar, dass nicht wenige dieser zweiten Generation ein ambivalentes, um nicht zu sagen gestörtes Verhältnis zu Deutschland, dem Land ihrer Kindheit und damit ihrer Sozialisation, hatten. Deshalb stellt sich auch hier die Frage, ob sich innerhalb dieser Generation so etwas wie kulturelle Heimat und eine wie auch immer geartete Identität entwickeln konnte. Und wenn, trifft das für alle Gruppen gleichermaßen zu, d.h. für die Kinder von deutschen Juden ebenso wie für die von polnischen, baltischen, galizischen oder ungarischen Juden, die seither - durch einen Zentralrat vertreten - „die Juden in Deutschland“ repräsentierten?

Bereits 1952 gab es in Westdeutschland über 100 Neugründungen jüdischer Gemeinden und zwei Synagogenneubauten (Saarbrücken und Stuttgart). Gemeindezentren wie das 1959 in der Berliner Fasanenstraße eingeweihte, das an der Stelle der ehemaligen Synagoge gebaut wurde, zeugten von einer vorsichtigen äußeren Konsolidierung. Innere Stärke spiegelte dies nicht. Das Modell der so genannten Einheitsgemeinde, mit dem sämtliche jüdische Strömungen von der Orthodoxie bis zum Reformjudentum unter ein und dasselbe Dach kamen,

1 Interview mit Paul Spiegel. Zitiert nach: Richarz, Monika: Leben in einem gezeichneten Land. Juden in Deutschland nach 1945. In: Die Geschichte der Juden in Deutschland. Hrsg. von Arno Herzig und Cay Rademacher. Hamburg 2007. S. 243. 
bot häufig die einzige Chance, noch als organisierte jüdische Gemeinschaft vor Ort zu überleben. Auch das System der Sozialfürsorge und Altenpflege wurde ausgebaut - ein wachsendes Erfordernis in Anbetracht zunehmender Überalterung und demographischer Stagnation. Auch die Gründung von einigen Jugendzentren und jüdischen Volkshochschulen konnte nicht darüber hinwegtäuschen, dass sich das organisierte Judentum in Deutschland - Jahrzehnte nach der Schoa - auf seine endgültige Auflösung zubewegte.

Bei der Grundsteinlegung des 1986 vollendeten Gemeindezentrums in Frankfurt am Main bemerkte der damalige Architekt und spätere stellvertretende Vorsitzende des Zentralrats der Juden in Deutschland, Salomon Korn, zwar: „Wer ein Haus baut, will bleiben“ ${ }^{2}$ - doch wer konnte sich langfristig überhaupt noch einrichten, und wie?

Bei genauerer Betrachtung zeigte sich aber, dass das Interesse vieler Gemeindemitglieder an der jüdischen Religion abnahm und jüdische Tradition wie Kultur nur noch bruchstückhaft an die nächste Generation weitergegeben wurden. Ähnlich wie in vielen christlichen Gemeinden beschränkte sich der Besuch der Gottesdienste nun auf die Hohen Feiertage, und die religiösen Riten wurden eher aus traditioneller Verbundenheit denn aus religiöser Überzeugung praktiziert. Die wenigsten Juden lebten und leben seither nach den jüdischen Religionsgesetzen. Als gemeinsamer Konsens ergab sich indes die Erinnerung an nationalsozialistische Verfolgung und Schoa, der Kampf gegen neue Formen von Antisemitismus und Rassismus wie auch eine starke Solidarität mit Israel.

Dennoch machten sich manche innerjüdischen Gegensätze weiterhin an religiösen Fragen fest. Verstand sich beispielsweise bis vor 1933 ein Großteil der deutschen Juden als liberal und war Mitglied einer jüdischen Reformgemeinde, so waren die Neugründungen der Einheitsgemeinden nach 1945 vornehmlich orthodox ausgerichtet, was auch innerhalb der Gemeinden zu Konflikten führte. Denn den orthodoxen Ritus führten die Rabbiner ein, die ausschließlich aus dem Ausland kamen. Eine Rabbinerausbildung innerhalb Deutschlands gab es nach 1945 nicht mehr und das liberale Judentum, das mit Leo Baeck, Gunther Plaut und anderen Deutschland in der NS-Zeit verlassen hatte, schaute zunächst verhalten auf das sich neu formierende jüdische Leben. Es sollte bis Anfang der 1990er Jahre dauern, bis es in Anknüpfung an die Vorkriegszeit wieder eine liberale Rabbinerausbildung in Deutschland gab - ein Zeichen für den wachsenden Bedarf an Rabbinern und der sich abzeichnenden religiösen Diversifizierung innerhalb der jüdischen Gemeinden.

2 Vgl. Korn, Salomon: Geteilte Erinnerung. Beiträge zur deutsch-jüdischen Gegenwart. Berlin 2001. S. 14. 
Mit dem Fall der Mauer und der Wiedervereinigung Deutschlands vollzog sich dann eine vollständige Transformation des jüdischen Lebens. Diese Transformation zeichnete sich zuvor weder durch den hüben wie drüben in vielen Gemeinden vollzogenen Generationenwechsel innerhalb der Gemeindeführung ab, noch durch eine kollektive Übersiedlung der kaum mehr als 400 Juden, die sich in der DDR noch auf acht Gemeinden verteilten.

Vielmehr bewirkte der Fall des Eisernen Vorhangs, dass seither über 200.000 Juden - einschließlich ihrer nichtjüdischen Verwandten - vom Territorium der früheren Sowjetunion in das wiedervereinigte Deutschland gekommen sind. Binnen kürzester Zeit entstanden neue Gemeinden, in Regionen, die bisher keinerlei jüdische Infrastruktur besaßen. In den bestehenden Gemeinden wie Berlin, Frankfurt, München vervielfachten sich die Mitgliederzahlen, neue Synagogen und Gemeindezentren entstanden quer durch das Land.

Der Zuzug der „russischen Juden“ - wie sie in stark vereinfachter Form in der Migrationsforschung bezeichnet werden - hat das Judentum in Deutschland tatsächlich vor dem demographischen Kollaps gerettet, zumindest auf einige Jahrzehnte hinaus. ${ }^{3}$ Insofern war es kaum verwunderlich, dass die in Deutschland schon wieder länger ansässigen Juden diesen Zuzug als große Chance begriffen und die Einwanderung auch von Teilen der nichtjüdischen Öffentlichkeit ausdrücklich begrüßt wurde. In der Anfangseuphorie wurde dagegen kaum wahrgenommen, dass eine erfolgreiche Integration der „Neuen“ auch eine enorme inter-kulturelle Akzeptanz und ein umfassendes Kennenlernen und gegenseitige Verständnis voraussetzen würde.

Wie wir heute wissen, kamen die Juden aus der einstigen UdSSR und ihre häufig nichtjüdischen Ehepartner und Angehörigen mit sehr unterschiedlichen Erwartungen und Lebenseinstellungen nach Deutschland, Israel und in die USA: Der Grad der Säkularisierung war unter ihnen fraglos weit stärker vorangeschritten als unter Juden in West- und Mitteleuropa. Viele betrachten dies bis heute als kein wirkliches Problem. Sie definieren ihr Judentum vielmehr über ethnische Zugehörigkeit, Antisemitismuserfahrungen, ausgeprägte Intellektualität, Interesse für jüdische Geschichte und Philosophie und häufig auch über eine Mischung aus Elementen russischer und jüdischer Kultur.

Während der 1990er Jahre, in denen sich die russischen Juden in Deutschland anschickten, die eindeutige demographische Mehrheit in den meisten lokalen jüdischen Gemeinden zu „erringen“, waren viele von ihnen zunächst noch mit

3 Vgl. hierzu u.a. Ein neues Judentum in Deutschland? Fremd- und Eigenbilder der russisch-jüdischen Einwanderer. Hrsg. von Julius H. Schoeps, Willi Jasper u. Bernhard Vogt. Potsdam 1999 (Neue Beiträge zur Geistesgeschichte 2); Ausgerechnet Deutschland! Jüdisch-russische Einwanderung in die Bundesrepublik. Hrsg. von Dmitri Belkin u. Raphael Gross. Frankfurt a.M. 2010. 
elementaren Fragen der sozialen und gesellschaftlichen Integration in die deutsche Gesellschaft beschäftigt. Die älteren Zuwanderer sahen sich häufig außerstande, noch die deutsche Sprache zu erlernen, und blieben zwangsläufig meist unter sich. In Großstädten wie Berlin fanden viele von ihnen aber Rückhalt in autarken Netzwerken und Milieus, die die Berliner Sozialwissenschaftlerin Judith Kessler in den 1990er Jahren als eine Art „russische Kolonien“ beschrieben hat. ${ }^{4}$

Doch auch die mittlere Generation der russischen Juden - zumindest jene, die das 40. Lebensjahr erreicht hatten - machten häufig die Erfahrung gesellschaftlicher Marginalisierung, oft primär bedingt durch einen gravierenden beruflichen Statusverlust.

Waren diese Menschen in Odessa, Wolgograd oder Vilnius angesehene Ärzte, Professoren oder Literaten, so standen sie nun als auf dem Arbeitsamt nicht vermittelbar in Schlangen vor den Jobagenturen. Hinzu kamen - und dies kennen die Kollegen in Israel von ihren Studien her ganz genauso - gravierende kulturelle Differenzen zur Aufnahmegesellschaft, sei dies in Bezug auf bevorzugte Sprache, Literatur, Musik, Theater, Mode oder Pädagogik. ${ }^{5}$ Beiderseitige Irritationen waren die Folge. Interessanterweise haben aber die allerwenigsten russisch-jüdischen Zuwanderer einen Bedarf gesehen, ihre Herkunftskultur einer rascheren sozialen Integration zu opfern oder sie gar zu verleugnen. Das zeugt insgesamt von einem starken kollektiven Selbstbewusstsein - das auch in den jüdischen Gemeinden spürbar ist. Russische Juden empfinden sich zudem häufig als Teil einer „transnationalen Diaspora“, Gleichgesinnte leben auf mindestens drei Kontinenten.

Mit der Zuwanderung der russischen Juden nach Deutschland ist die jüdische Gemeinschaft hier nicht nur deutlich gewachsen, sie hat auch deutlich an Pluralität gewonnen. Das kann und sollte als Chance begriffen werden, was wiederum keine Selbstverständlichkeit ist.

Jüdische Kultur in Deutschland - bringt diese eine neue Identität hervor? Ist diese Fragestellung „nur“ einer Innen- oder auch einer Außenperspektive inhärent? Und sind dabei nur die Mitglieder der Jüdischen Gemeinden gemeint, oder vielleicht sogar nur diejenigen, die die jüdischen Gesetze einhalten? Oder gilt sie darüber hinaus auch für die Juden, die keiner Gemeinde angehören, oder gene-

4 Kessler, Judith: Identitätssuche und Subkultur. Erfahrungen der Sozialarbeit in der Jüdischen Gemeinde zu Berlin. In: Schoeps, Jasper u. Vogt (Hrsg.): Judentum (wie Anm. 3). S. 140-162; Kessler, Judith: Jüdische Immigration seit 1990. Resümee einer Studie über 4000 jüdische Migranten aus der ehemaligen Sowjetunion in Berlin. In: Zeitschrift für Migration und soziale Arbeit (1990), 1. S. 40-47.

5 Vgl. Glöckner, Olaf: Immigrated Russian Jewish Elites in Israel and Germany. Integration, SelfImage and Role in Community Building. Saarbrücken 2011. 
rell allen mit einem jüdischen Background - egal ob religiös, areligiös oder indifferent?

Jedenfalls erleben wir innerhalb der jüdischen Communities in Deutschland seit den späten 1990er Jahren eine wachsende religiöse wie kulturelle Ausdifferenzierung, ohne dass das Modell der „Einheitsgemeinde“ prinzipiell aufgegeben wurde. Es gibt heute gleichwohl wieder eine Reihe von selbstständigen liberalen Gemeinden, in Ansätzen wieder ein konservatives Judentum (Masorti - v.a. in Berlin), und selbst auf der gesetzestreuen, orthodoxen Seite gibt es - wenn auch in Form eher kleinerer Gemeinschaften - dynamische Neuerungen. Eher säkular orientierte Juden bilden ihre Netzwerke über jüdische Kultur- und Bildungsvereine, Theater- und Musikfestivals und manchmal auch über politische Initiativen und Projekte. Selbstverständlich sind russische Juden - neben den einheimischen Juden - in all diesen Zusammenschlüssen vertreten.

Heute, rund zwei Jahrzehnte nach dem Beginn der großen russisch-jüdischen Zuwanderung, gewinnen Fragen der kollektiven und individuellen Identität, der Selbstverortung und der kulturellen Orientierung wieder deutlich an Gewicht. Doch erlaubt man sich nochmals einen historischen Rückblick, ergibt sich im Vergleich Erstaunliches: Deutschlands Juden flüchteten während der 1930er Jahre aus ihrem Land - und nahmen quasi ihr deutsch-jüdisches Kulturerbe mit. Russlands Juden erlebten eine systematische und allmähliche Zerstörung ihrer Einrichtungen und Traditionen während der 70-jährigen Sowjetdiktatur. Schon kurz nach der Bolschewistischen Revolution war Hebräisch verboten, Stalin zerstörte die jiddische Kultur zu weiten Teilen. Religiöses Gemeinschaftsleben beschränkte sich dann in den letzten Jahrzehnten des Sowjetstaates nur noch auf einige wenige Synagogen - Gorbatschows Liberalisierung kam auch in dieser Hinsicht zu spät.

Dennoch haben viele Juden aus der Sowjetunion ihr Judentum nicht vergessen oder negiert. In der zweiten Generation wird sich nun entscheiden, wie sie mit ihrem Erbe langfristig umgehen - und gerade auf das jüdische Leben in Deutschland wird dies essentielle Auswirkungen haben. Dass die russischen Juden allerdings einen Anschluss an hiesige Traditionen - wie sie beispielsweise im Schaffen und Wirken von Moses Mendelssohn, Leo Baeck, Martin Buber oder Bertha Pappenheim zu finden sind - suchen werden, darf bezweifelt werden.

Eine sehr spannende Frage ist, inwiefern die zweite Generation der russischen Juden gemeinsame kulturelle - oder auch religiöse - Identitäten mit den Kindern der in Deutschland schon länger ansässigen Juden entwickeln wird und welche Rolle schließlich die Nachfahren der ebenfalls noch im Lande lebenden, wenn auch zahlenmäßig sehr wenigen deutschen Juden (,Jeckes“) in Zukunft spielen können. 
Wo wird es um Kontinuitäten in der jüdischen Religion gehen - und wenn, mit welchen Präferenzen? Welches Selbstverständnis entwickeln Jüdische Gemeinden, in denen die Zahl säkularer Mitglieder wächst? Wo vernetzen sich säkulare Juden außerhalb der Gemeinden? Diese Fragen kommen zwangsläufig auf, wenn eine Verortung der Juden in Deutschland und ihre Identitäten und möglichen Identitätswechsel, ihre Eigen- und Fremdzuschreibungen in den Fokus rücken.

Schließlich soll hier auch nicht die wachsende Zahl von Israelis unerwähnt bleiben, welche langfristig oder permanent in Deutschland leben und deren Zahl heute allein in Berlin auf etwa 20.000 geschätzt wird. Berlin ist seit der deutschen Wiedervereinigung zu einem stetigen Magneten für junge Leute aus Israel geworden, keineswegs nur für Studenten, sondern zunehmend auch für Künstler, Unternehmer, Akademiker und andere.

All diese Gruppen, die „Alteingesessenen“, die „DPs“, die „Gesetzestreuen“, die „Russen“ und neuerdings die „Israelis“ bilden keine homogene Einheit. Ihre religiösen, politischen oder kulturellen Bekenntnisse sind ebenso unterschiedlich wie ihre individuellen Erlebnisse, Erfahrungen und Wertvorstellungen. Aber - nochmals die Frage -, wie leben und definieren sie sich als Juden in Deutschland?

Begeben sie sich bewusst in die genannten Subkulturen und Binnengesellschaften oder werden sie durch die Umgebungsgesellschaft in diese genötigt? Besteht bei den einen wie den anderen der Wunsch nach Integration in die Mehrheitsgesellschaft oder ziehen sie eine Parallelgesellschaft vor? Und rein rhetorisch gefragt: Wird man je von einer gemeinsamen Identität von Juden in Deutschland sprechen können?

Zurück zur Metapher des Koffers. Was befand sich neben den persönlichen Dokumenten, der Kleidung und Erinnerungsstücken in den Koffern, die die Migranten, ob nach 1933, nach 1945 oder nach 1989 in ein neues Leben - eine neue Identität - mitnahmen? In Buenos Aires - die südamerikanische Metropole beherbergt eine der größten jüdischen Gemeinden außerhalb Israels - lebt Robert(o) Schopflocher. Er floh 1937 mit seinen Eltern aus Deutschland, er war damals 14 Jahre alt. Auf die Frage: „Wie würden Sie sich selbst bezeichnen? Als Argentinischer Jude? Als argentinischer Jude mit deutschen Wurzeln? Als deutscher Jude in Argentinien?“" antwortete er umgehend. Aber er antwortete mit einem Gedicht, einem selbst verfassten, das er als etwas Achtzigjähriger zu Papier gebracht hatte, noch lange bevor er seine Autobiografie niederschrieb. ${ }^{6}$

Das Gedicht trägt den Titel Geständnis, das gleichwohl auch als Bekenntnis und Selbstverortung gewertet werden kann:

6 Schopflocher, Robert: Weit von Wo - mein Leben zwischen drei Welten. München 2010. 


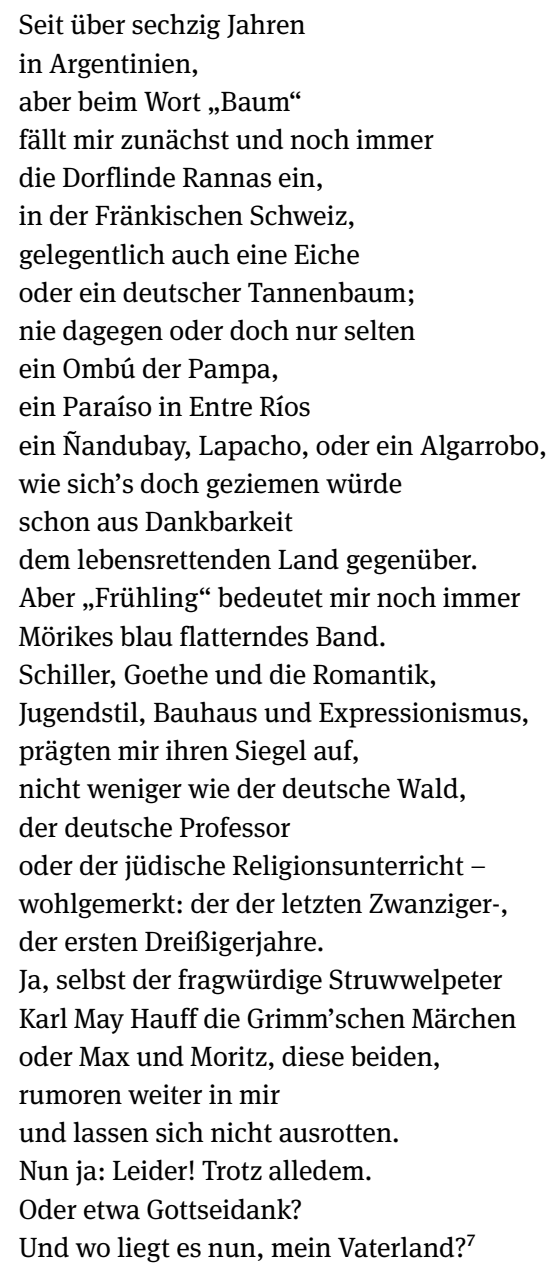

Was bringt dieses Gedicht zum Ausdruck? Und was bewog den Verfasser zu diesen Zeilen? Schopflocher lebt seit nunmehr über 75 Jahren in Argentinien, hat dort eine deutschstämmige Jüdin geheiratet, zwei Söhne großgezogen, die er in drei Kulturen erzog, in der argentinischen, der jüdischen und in der deutschen. Er nimmt aktiv am jüdischen Gemeindeleben in Buenos Aires teil, geht in eine Synagoge nach liberalem Ritus (wo bis vor wenigen Jahren der Gottesdienst auf Deutsch gehalten wurde). Und er lebt, wie er selbst betont, noch immer in drei Welten, in denen er jeweils eine „Heimat“ gefunden hat.

7 Schopflocher, Robert: Hintergedanken. Gedichte aus zwei Jahrzehnten. Nürnberg 2012. S. 33. 
Lassen sich in derartigen Lebensläufen und Lebensentwürfen emigrierter deutschsprachiger Juden auch Parallelen zu heute in Deutschland lebenden Juden aus der ehemaligen Sowjetunion finden? Schopflochers kulturelle wie religiöse Sozialisation erfolgte in Deutschland, als Jugendlicher kam er nach Argentinien, erlernte die spanische Sprache, studierte und arbeitete den äußeren Umständen geschuldet in einem Beruf (in der Landwirtschaft), der ihm nicht lag. Lebte in politischen Verhältnissen, die nicht seiner Überzeugung entsprachen, und doch richtete er sich ein in diesem Leben, dem Land, das ihm und seiner Familie Unterschlupf bot, das ihm aber auch die Möglichkeit offerierte, sein Leben selbst $\mathrm{zu}$ gestalten und seine verschiedenen Identitäten $\mathrm{zu}$ finden bzw. zu bewahren.

Hier liegt möglicherweise aber auch eine Crux im Vergleich zwischen Schopflocher und einem Ukrainer, Letten oder Usbeken, Moskauer oder Leningrader ähnlichen Alters. Beide zeichnen sich im Verlaufe ihres Lebens durch unterschiedliche Erfahrungshorizonte aus, was sich nicht zuletzt auch in unterschiedlichen Wahrnehmungen und kulturellen Codes widerspiegelt. Um nur ein Beispiel aus dem Bereich der Gedenkkultur zu wählen: Während Roberto Schopflocher den 9. November 1938 als eines der markantesten Daten für das deutsch-jüdische Verhältnis erinnert, ist es für den ukrainischen, russischen oder litauischen Juden in Berlin, Leipzig oder München der 9. Mai 1945, dem er alljährlich gedenkt und zu dessen Anlass er seine von der Sowjetarmee verliehenen Orden trägt.

„Wenn ein Mensch - und eine Gesellschaft - nur das zu erinnern im Stande ist, was als Vergangenheit innerhalb der Bezugsrahmen einer jeweiligen Gegenwart rekonstruierbar ist, dann wird genau das vergessen, was in einer solchen Gegenwart keine Bezugsrahmen mehr hat. “8 In Anlehnung an Jan Assmanns Definition der bereits 1925 von dem Soziologen Maurice Halbwachs formulierten These stehen hier unterschiedliche Gedächtnisse aufgrund von unterschiedlichen Bezugsrahmen und Erfahrungshorizonten nebeneinander, die für den jeweils anderen nur schwer nachvollziehbar sind. Ein gemeinsames kollektives Gedächtnis lässt sich daraus kaum formen. Wichtig ist daher aber, dass es von allen Seiten eine Bereitschaft gibt den jeweils anderen Bezugsrahmen zu billigen und nicht auszugliedern.

Und doch gibt es frappierende Beispiele für eine kulturelle Synthese - gerade unter jungen Juden in Deutschland heute, die mittlerweile zur vierten Generation zählen. Ich möchte hier nur kurz darauf eingehen, dafür aber - wie ich finde - mit einen prägnanten Beispiel: Lena Gorelik, 1981 in Leningrad (Sankt Petersburg) geboren, kam 1992 mit ihrer Familie als russisch-jüdischer Kontingentflüchtling

8 Assmann, Jan: Das kulturelle Gedächtnis. Schrift, Erinnerung und politische Identität in frühen Hochkulturen. 5. Aufl. 2007. S. 36. 
nach Deutschland. In München ging sie zur Schule, wurde an der Deutschen Journalistenschule zur Journalistin ausgebildet und absolvierte anschließend ein Studium der Osteuropastudien an der Ludwig Maximilian Universität. 2004 veröffentlichte sie ihren ersten Roman (Meine weißen Nächte), es folgten drei weitere. Im letzten Jahr folgte ein Essayband mit dem Titel „Sie können aber gut Deutsch!“ Warum ich nicht mehr dankbar sein will, dass ich hier leben darf, und Toleranz nicht weiterhilft. Dieses schon im Titel provokante Statement versteht sie als eine Antwort auf die immer wieder gestellten Fragen, wie sie sich als ,jüdische“ „Asylantin“ in Deutschland fühle. Über ihr „Jüdischsein“ bemerkte sie 2007 in einem Interview: „In Deutschland ist es etwas, womit ich mich auseinander setzen muss, aber nicht, weil ich es will, sondern weil ich dazu gebracht werde. Für mich persönlich ist Jüdischsein ein Gefühl. Dazu gehört diese bestimmte Art von Humor und der Lebenswahrnehmung. “9

Das aktuelle Buch, so betont sie darin, habe sie „über Menschen“ in Deutschland geschrieben:

\footnotetext{
Über Menschen, die in diesem Land leben, es in irgendeiner Weise beeinflussen, bereichern, verwirren, es letztlich zu dem machen, was es ist.

Denn, nachdem wir mehrere Jahrzehnte zu spät entdeckt haben, dass wir doch schon ziemlich lange ein Einwanderungsland sind und die Debatte (denn wir Deutschen lieben Debatten!) darüber, was das bedeutet, irgendwie verpasst zu haben und nun schwer damit beschäftigt sind, „,in unserer Mitte angekommene türkischstämmige Mitbürger“ in PolitTalkshows und zu Integrationsbündnissen einzuladen, damit sie uns endlich einmal sagen, wie sie und ihresgleichen sich in unsere nicht definierte und wahrscheinlich auch nicht definierbare deutsche Gesellschaft integrieren können, haben wir vergessen, dass wir dabei vor allem über Menschen sprechen. ${ }^{10}$
}

Die Zukunft wird zeigen, ob in weiteren drei bis vier Generationen Juden in Deutschland in einer oder mehreren Welten leben, oder, wie es Lena Gorelik bereits heute pointiert definiert, „die in unserer Mitte angekommenen türkischstämmigen Mitbürger“ „uns Deutschen“ endlich erklärten, wie „sie und ihresgleichen“ sich in „unsere“ deutsche Gesellschaft „integrieren können“.

Eine mögliche Antwort auf die eingangs gestellten Fragen mag Lena Gorelik damit gegeben haben. Aber entspringt diese einer jüdischen Kultur in Deutschland?

9 Die Welt, 7.4.2007.

10 Gorelik, Lena: „Sie können aber gut Deutsch!“: Warum ich nicht mehr dankbar sein will, dass ich hier leben darf, und Toleranz nicht weiterhilft. München 2012. S. 12. 